\title{
Fuzzy Multi-Objective Linear Programming and Simulation Approach to the Development of Valid and Realistic Master Production Schedule
}

\author{
IIham Supriyanto MSc., Prof. Dr. Ing. Bernd Noche \\ Universität Duisburg - Essen \\ Institut Transportsysteme und -Logistik
}

\begin{abstract}
Master production schedule (MPS) plays an important role in an integrated production planning system. It converts the strategic planning defined in a production plan into the tactical operation execution. The MPS is also known as a tool for top management to control over manufacture resources and becomes input of the downstream planning levels such as material requirement planning (MRP) and capacity requirement planning (CRP). Hence, inappropriate decision on the MPS development may lead to infeasible execution, which ultimately causes poor delivery performance. One must ensure that the proposed MPS is valid and realistic for implementation before it is released to real manufacturing system. In practice, where production environment is stochastic in nature, the development of MPS is no longer simple task. The varying processing time, random event such as machine failure is just some of the underlying causes of uncertainty that may be hardly addressed at planning stage so that in the end the valid and realistic MPS is tough to be realized. The MPS creation problem becomes even more sophisticated as decision makers try to consider multi-objectives; minimizing inventory, maximizing customer satisfaction, and maximizing resource utilization. This study attempts to propose a methodology for MPS creation which is able to deal with those obstacles. This approach takes into account uncertainty and makes trade off among conflicting multi-objectives at the same time. It incorporates fuzzy multi-objective linear programming (FMOLP) and discrete event simulation (DES) for MPS development.
\end{abstract}

Keyword: Master Production Schedule, Fuzzy Multi-Objective Linear Programming, ERP, Discrete Event Simulation, Production Planning

\section{Introduction}

According to American Production and Inventory Control Society (APICS), MPS is the declaration of what the company expects to manufacture in terms of configuration, quantities, and specific dates that drives MRP and other subsequent activities of a manufacturing company. Due to its vital role in production planning system, it is necessary to ensure that MPS is valid and realistic; otherwise the company may be unresponsive to customer needs or wasteful in its use of resources.

Proud argues that the real challenge of MPS creation is to balance available and requirement capacity [Pro99]. In this context, the viable solution may be easily found 
for deterministic capacity, however, it turns into intricate problem if both available and requirement capacities are stochastic or fuzzy. It is a fact that the available capacity of productive resource cannot be determined precisely due to unpredictable events such as unplanned breakdown, labor shortage, or material shortage. The requirement capacity for producing demand is also fuzzy because of varying processing time, setup time, or queue time.

In practice, it seems that this phenomenon is not taken into account by the modern ERP system. Rough-cut capacity planning (RCCP) function, which provides the productive resource profile for MPS, is not designed to deal with uncertainty. RCCP even considers only key or critical resources. Whereas, CRP function, which is expected to give a more detailed capacity check, is also neglecting uncertainty and even it performs only infinite forward loading instead of finite capacity analysis. It is in this sense that the current MPS system is limited to answering the question: Do we have a chance to meet the production plan? And do we have a chance to meet the master schedule?

Concerning to this issues, several studies have suggested a verification process to check the validity of tentative MPS. Higgins et al. have proposed a simulation of "What-if" analysis to be executed on the tentative MPS in order to find an optimum and realistic MPS [Hig92]. Kochhar et al. have developed a knowledge-based system approach, which is combining human expertise with computer computation, to achieve an accurate and realistic master schedule [Koc98]. Heizer et al. shared an idea about iterative planning process which allows a planner to check the validity of each planning process [Hei06].

In addition to the substitution of verification process, researchers also employ various advanced optimization technique in order to enhance MPS quality. For instance, Vieira et al. applied simulated annealing to solve MPS problem [Vie04]. This study reveals some drawbacks of simulated annealing such as overcoming local optimum. Soares et al. introduced new genetic algorithm structure for solving MPS problem [Soa08]. It formulates the fitness function which aims to minimize inventory level, maximize service level, minimize overtime and minimize inventory level below safety stock. At the end, Vieira et al. has compared genetic algorithms and simulated annealing for master production scheduling problems [Vie03].

The reviewed approaches have given worthy contribution to the development of MPS, especially to balance requirement capacity and available resource in effective manner while maintaining trade-off among the conflicting objectives. Only one thing needs to be improved is the underlying assumption of those approaches which ignore the capacity uncertainty. In order to bridge the gap, this research proposes a methodology, which employs FMOLP to model mathematical fuzzy objectives and constraints as well as DES to capture the dynamic behavior of manufacturing system and to verify the tentative MPS. 


\section{Iterative Production Planning}

In order to ensure successful operation execution, the valid and realistic MPS must be developed [She03]. In this context, term valid means that the available capacity should be equal to the required capacity and the material due date is equal to the material need dates [Pro99]. Whereas, realistic means that the MPS must be feasible to implement and able to deliver the defined planning goal or decision maker (DM) targets. According to Sheikh, the development of actual scheduling is usually iterative, with a preliminary schedule being drawn up, checked for problems, and revised [She03]. After a schedule has been determined, some points should be checked: Does the schedule feasible to implement? Does the schedule meet the demand forecast? Does the schedule provide for flexibility and backups in case disturbance occurs? and so on. Problem in any one of these areas may force a revision of the schedule and a repeat of the previous step. The iterative process continues until all questions are answered and the planning goal is met.

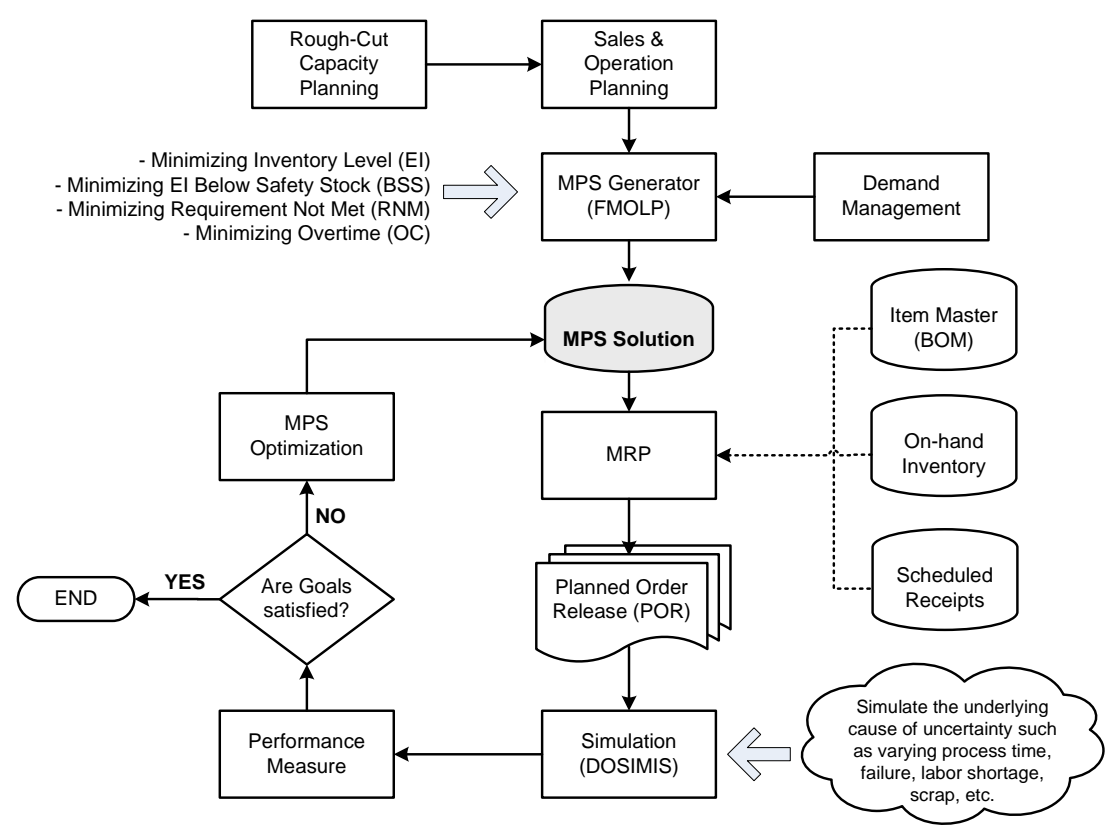

Figure 1: Schema of iterative MPS development

This study employs the same concept as the idea but using DES as a tool for the verification of master schedule. Figure 1 depicts a scheme for the creation of valid and realistic MPS proposed in this study. The demand management supplies all of the demand requirements and customer orders to MPS generator. Likewise, the sales and operation planning (SOP) indicates the operating constraints within which MPS function must work. MPS generator produces a tentative MPS solution that satisfies DM's target in terms of inventory level (EI), requirement not-met (RNM), inventory below safety Stock (BSS) and overcapacity (OC). Based on the given MPS, MRP function generates a set of planned order request (POR) by considering bill of material (BOM), on-hand inventory and scheduled receipts. POR is work document which contains information associated with production such as part number, quantitiy to be manufactured, work center, production start date, due date, and customer. To 
verify the validity of master schedule, the execution of PORs is simulated under various stochastic environments. The MPS is considered as valid and realistic only if the simulation output satisfies the defined DM's goals.

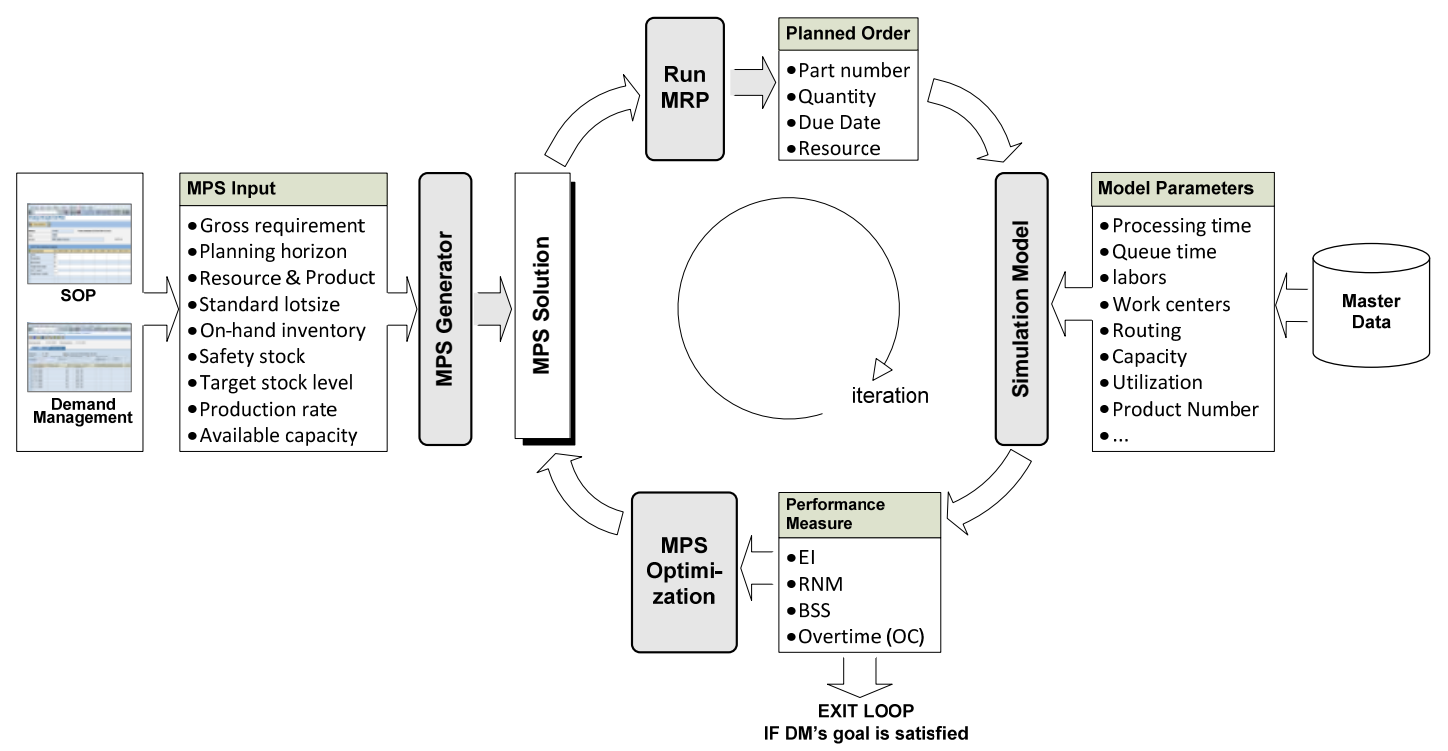

Figure 2: The information flow of MPS development

In order to realize the concept, an integrated planning system consisting of three commercial software (SAP, Matlab, and Dosimis-3) is developed. Figure 2 denotes the information flow of MPS development. To keep the consistency of planning logic, all production planning functions are executed in SAP except MPS. The MPS solution is generated separately in Matlab by adopting FMOLP technique whose formulation is explained in details in chapter 3. The input parameters (i.e. gross requirement, lot-size, safety stock, etc) described by Vieira et al. are automatically retrieved from SAP [Vie04]. Using those inputs, a tentative MPS is generated and subsequently entered into SAP as input of MRP. Simultaneously, Dosimis-3 retrieves master data (i.e. workstation, routing, processing time, capacity, etc.) from SAP to build simulation model. Ziems et al. have described step-by-step procedure to develop simulation model using Dosimis-3 [Zie93]. Then, the outcome of simulation is sent to Matlab. The key performance indicators are calculated and compared to the initial planning goal. If the DM's goals are not satisfied, the given MPS will be reoptimized and verified again. This process is repeated until the DM's goal is achieved.

\section{MPS Generator}

According to Vieira et al. [Vie03] and Soares et al. [Soa08], the MPS problem can be mathematically modeled as a mixed integer program as follow.

$$
\text { Minimize EI }=\sum_{k=1}^{K}\left(\frac{\sum_{p=1}^{p} E I_{k p}}{T H}\right) \quad \text { Minimize } O C=\sum_{r=1}^{R} \sum_{p=1}^{P} O C_{r p}
$$


Minimize BSS $=\frac{\sum_{k=1}^{K} \sum_{p=1}^{P} B S S_{k p}}{T H}$

Minimize $R N M=\frac{\sum_{k=1}^{K} \sum_{p=1}^{P} R N M_{k p}}{T H}$

Where,

$$
\begin{array}{ll}
C U H_{r p}=\sum_{k=1}^{K} \frac{\left(B S_{k p} B N_{k p}\right.}{U R_{k r}} & M P S T_{k p}=\sum_{r=1}^{R} M P S_{k p r} \\
E I_{k p}=\max \left[0,\left(M P S_{k p}+B I_{k p}\right)-G R_{k p}\right\rfloor & M P S_{k p r}=B N_{k p r} \times B S_{k p r} \\
B S S_{k p}=\max \left[0,\left(S S_{k p}-E I_{k p}\right)\right\rfloor & \\
R N M_{k p}=\max \left[0,\left(G R_{k p}-\left(M P S T_{k p}+B I_{k p}\right)\right)\right] & \\
O C_{r p}=\max \left[0,\left(C U H_{r p}-A C_{r p}\right)\right\rfloor & \\
B I_{k p}= \begin{cases}O H_{k} & s e(p=1) \\
E I_{k(p-1)} & s e(p>1)\end{cases} &
\end{array}
$$

When all goals are fuzzy and each of them has aspiration level $Z^{*}(i=1,2,3,4)$, the above crisp model is transformed into fuzzy statement as follow.

Find $x=\left\lfloor x_{111}, x_{112}, x_{113}, \ldots, x_{k p r}\right\rfloor$ to satisfy

$$
\begin{aligned}
& \tilde{Z}_{1}(x)=E \tilde{I}(x)=\sum_{k=1}^{K}\left(\frac{\sum_{p=1}^{p} E I_{k p}}{T H}\right)<\sim Z_{1}^{*} \\
& \tilde{Z}_{2}(x)=R N \tilde{M}(x)=\frac{\sum_{k=1}^{K} \sum_{p=1}^{P} R N M_{k p}}{T H}<\sim Z_{2}^{*} \\
& \tilde{Z}_{3}(x)=B S \tilde{S}(x)=\frac{\sum_{k=1}^{K} \sum_{p=1}^{P} B S S_{k p}}{T H}<\sim Z_{3}^{*} \\
& \tilde{Z}_{4}(x)=O \tilde{C}(x)=\sum_{r=1}^{R} \sum_{p=1}^{P} O C_{r p}<\sim Z_{4}^{*}
\end{aligned}
$$

Subject to

$$
g_{2}(x)=C U H_{r p}-A C_{r p} \leq O L_{\max } \quad ; x_{k p r} \geq 0
$$

Where $O L_{\max }$ represents maximum overtime allowed for each period. Assuming that $Z^{0}{ }_{i}$ and $Z_{i}{ }_{i}$ denote the value of the objective function $Z_{i}(i=1,2,3)$ such that the degrees of membership function is 0 or 1 respectively. The linear function of the membership function for objectives $Z_{1}, Z_{2}, Z_{3}$ is described as follow. 


$$
\mu_{Z i}(x)= \begin{cases}0 & Z_{i}(x) \geq Z_{i}^{1} \\ \left(Z_{i}^{1}-Z_{i}(x)\right) /\left(Z_{i}^{1}-Z_{i}^{0}\right) & Z_{i}^{0} \geq Z_{i}(x) \geq Z_{i}^{1} \quad ; i=1,2,3 \\ 1 & Z_{i}(x) \leq Z_{i}^{0}\end{cases}
$$

When $a$ and $b$ denotes the limit of the admissible violation of overcapacity, the triangular membership function for objective $Z_{4}$ is written as follow.

$$
\mu_{Z 4}(x)= \begin{cases}1-\left(Z_{4}^{1}-Z_{4}(x)\right) / a & Z_{4}^{1}-a \leq Z_{4}(x) \leq Z_{4}^{1} \\ 1-\left(Z_{4}(x)-Z_{4}^{1}\right) / b & Z_{4}^{1} \geq Z_{4}(x) \geq Z_{4}^{1}+b \\ 0 & \text { else }\end{cases}
$$

Using the convex fuzzy model proposed by Bellman et al. [Bel70] and Sakawa [Sak93], the equivalent crisp model for fuzzy formulation (eq.2) can be expressed as the following linear programming.

$\max w_{1} \mu_{Z 1}+w_{2} \mu_{Z 2}+w_{3} \mu_{Z 3}+w_{4} \mu_{Z 4}$

subject to.

$$
\begin{aligned}
& \mu_{Z 1} \leq\left(Z_{1}^{1}-Z_{1}(x)\right) /\left(Z_{1}^{1}-Z_{1}^{0}\right) \\
& \mu_{Z 2} \leq\left(Z_{2}^{1}-Z_{2}(x)\right) /\left(Z_{2}^{1}-Z_{2}^{0}\right) \\
& \mu_{Z 3} \leq\left(Z_{3}^{1}-Z_{3}(x)\right) /\left(Z_{3}^{1}-Z_{3}^{0}\right) \\
& \mu_{Z 4} \leq 1-\left(Z_{4}(x)-Z_{4}^{1}\right) / b \\
& \mu_{Z 4} \leq 1-\left(Z_{4}^{1}-Z_{4}(x)\right) / a \\
& C U H_{r p}-A C_{r p} \leq O L_{\max } \\
& w_{1}+w_{2}+w_{3}+w_{4}=1 \\
& \mu_{Z i} \in[0,1] ; x_{k p r} \geq 0 ; i=1,2,3,4 \quad r=1,2,3, \ldots ; p=1,2,3, \ldots
\end{aligned}
$$

Where $w_{i}$ denotes the weighting coefficients that show the relative importance among the fuzzy objectives. The MPS solution $x^{*}$ is obtained by solving the above crisp model using genetic algorithm.

\section{$4 \quad$ MPS Optimization}

Several symptoms of mismanaged MPS can be identified from excessive unplanned overtime or overcapacity, extensively front - loaded capacity plan, excessive past due shop orders and increasing component inventory [She03, Pro99]. In this study, those symptoms will be used to justify the feasibility of tentative MPS. The optimization is carried out immediately as one of them is recognized during simulation. For this purpose, the fuzzy-based load leveling model is developed. 
The principle of load leveling technique is simply to shift some quantities of product to be manufactured from the over-loaded resources to the under-loaded resources. However, this task becomes sophisticated as multiple resources, multiple products, and multiple periods are considered. One should determine how many quantities and which products should be shifted, from or to which resources, and from or to which period. Inappropriate decision may cost poor performance of other criteria. For instance, producing too much quantity in early planning period may decrease overtime during peak load but consequently lead to high inventory level. The task becomes more complex as the conflicting multi-objectives are involved. Fortunately, an advanced optimization technique such as FMOLP enables one to solve such intricate problem. The linear programming model for the load - leveling problem is formulated as follow.

$$
\min R \tilde{T}(y)=\max \left[0, O L-\sum_{k=1}^{K} \frac{y_{k}}{U_{k o}}\right] \quad \min \quad D \tilde{I}(y)=\sum_{k=1}^{K} s_{k u} \times y_{k}
$$

subject to.

$$
\begin{aligned}
& g_{1}(y)=\sum_{k=1}^{K} \frac{y_{k}}{U_{k u}} \leq R C \\
& y_{k} \leq x_{k o} ; y_{k} \geq 0 ; k=1,2, \ldots, K
\end{aligned}
$$

Where $g_{1}(y)$ is constraint to ensure that resource $R_{u}$ does not turn into overloaded after leveling. When the reduced capacities $[R T]$ and the quantity of defect products [DI] are fuzzy, the equivalent crisp model for equation 6 can be written as follow.

$\max \beta_{1} \mu_{R T}+\beta_{2} \mu_{D I}$

Subject to.

$$
\begin{aligned}
& \mu_{R T} \leq \frac{R T(y)-R T^{0}}{R T^{1}-R T^{0}} \quad \mu_{D I} \leq \frac{D I(y)-D I^{0}}{D I^{1}-D I^{0}} \\
& g_{1}(y)=\sum_{k=1}^{K} \frac{y_{k}}{U_{k u}} \leq R C \\
& y_{k} \leq x_{k o} \quad ; y_{k} \geq 0 \quad ; k=1,2, \ldots, K \\
& \mu_{R T \mid D I} \in[0,1] \quad ; \beta_{1}+\beta_{2}=1
\end{aligned}
$$

$\left[R T^{0}, R T^{1}\right]$ denote the lower and upper bound of aspiration level for the reduced load capacity, whereas $\left[\mathrm{DI}^{0}, \mathrm{DI}^{1}\right]$ indicate lower and upper bound of aspiration level for defect products. Those aspiration levels are obtained by solving the multi-objective problem as single objective using, each time, only one objective. Parameters $\left[\beta_{1}, \beta_{2}\right]$ indicate relative importance between fuzzy objectives and the degrees of membership function for each objective are as follow. 


$$
\begin{aligned}
& \mu_{R T}(y)= \begin{cases}1 & R T(y) \leq R T^{0} \\
\frac{R T(y)-R T^{0}}{R T^{1}-R T^{0}} & R T^{0}<R T(y)<R T^{1} \\
0 & R T(y) \geq R T^{1}\end{cases} \\
& \mu_{D I}(y)= \begin{cases}1 & D I(y) \leq D I^{0} \\
\frac{D I(y)-D I^{0}}{D I^{1}-D I^{0}} & D I^{0}<D I(y)<D I^{1} \\
0 & D I(y) \geq D I^{1}\end{cases}
\end{aligned}
$$

To obtain solution $y^{*}{ }_{k}(k=1,2, . . K)$, which is the quantities of products to be shifted to available resources, the above crisp equation is solved using genetic algorithm.

\section{Study Case}

In order to test and provide thorough illustration about the proposed methodology, a simplified production scenario is given and solved. Let's assume a typical manufacturing system which consists of four production resources (can be machines or production lines) and each of them has available capacity per period 8 hours. The

\begin{tabular}{|c|c|c|}
\hline Para & Notation & Value \\
\hline $\begin{array}{ll}\text { - } & \text { Number of products } \\
\text { - } & \text { Number of production lines } \\
\text { - } & \text { Planning horizon } \\
\text { - } & \text { Standard lot size } \\
\text { - } & \text { Average of production rate } \\
\text { - } & \text { Initial inventory } \\
\text { - } & \text { Safety stock level } \\
\text { - } & \text { Gross requirement } \\
\text { - } & \text { Available capacity } \\
\text { - } & \text { Maximum overall overtime } \\
\text { - } & \text { Allowed maximum } \\
\text { - } & \text { Aspiration Levels }\end{array}$ & $\begin{array}{c}K \\
R \\
P \\
B S_{k p} \\
U R_{r p} \\
\\
O H_{k} \\
S S_{k p} \\
G R_{k p} \\
A C_{r p} \\
A C_{t o t} \\
O L_{m a x} \\
E I \\
R N M \\
B S S \\
O C \\
W_{i} \\
\beta_{i} \\
S_{k u}\end{array}$ & $\begin{array}{l}4(\mathrm{P}-403, \mathrm{P}-404, \mathrm{P}-405, \mathrm{P}-406) \\
4 \text { (WCFXM01, WCFXM02, WCFXM03, WCFXM04) } \\
20 \text { days } \\
10 \text { unit for all products and all planning periods } \\
\text { Production rates for P-403, P-404, P-40, and P-405 } \\
\text { are } 100,150,200, \text { and } 400 \text { units/hrs respectively } \\
\text { Zero for all products } \\
200 \text { units for all products and all planning periods } \\
\text { Table } 2 \\
8 \text { hours/period for each resource } \\
4 \text { hours during planning horizon } \\
4 \text { hours/ period for each resource } \\
Z_{1}^{0}=50, \quad Z_{1}^{1}=5250 \\
Z_{2}^{0}=0, \quad Z_{2}^{1}=3200 \\
Z_{3}^{0}=10, \quad Z_{3}^{1}=2000 \\
a=b=Z_{4}^{1}=4 \\
w_{1}=0.3, \quad w_{2}=0.3, \quad w_{3}=0.3, \quad w_{4}=0.1 \\
\beta_{1}=0.7, \quad \beta_{2}=0.3 \\
0 \% \text { for all resources }\end{array}$ \\
\hline
\end{tabular}
detail MPS inputs and other production parameters are presented in Table 1.

Table 1: MPS Inputs and Production Parameters 


\begin{tabular}{c|cccc||c|cccc}
\hline Day & P- 403 & P- 404 & P- 405 & P- 406 & Day & P- 403 & P- 404 & P- 405 & P- 406 \\
\hline $\mathbf{1}$ & 1300 & 1250 & 1390 & 580 & $\mathbf{1 1}$ & 1400 & 1000 & 1610 & 680 \\
$\mathbf{2}$ & 930 & 1360 & 1360 & 530 & $\mathbf{1 2}$ & 340 & 1200 & 1460 & 690 \\
$\mathbf{3}$ & 1100 & 1660 & 1450 & 1650 & $\mathbf{1 3}$ & 420 & 320 & 1310 & 960 \\
$\mathbf{4}$ & 720 & 1490 & 1420 & 840 & $\mathbf{1 4}$ & 760 & 1440 & 1440 & 500 \\
$\mathbf{5}$ & 740 & 590 & 1270 & 230 & $\mathbf{1 5}$ & 880 & 1300 & 1070 & 280 \\
$\mathbf{6}$ & 1030 & 1520 & 1710 & 210 & $\mathbf{1 6}$ & 940 & 1030 & 1670 & 560 \\
$\mathbf{7}$ & 970 & 1570 & 1540 & 1710 & $\mathbf{1 7}$ & 1190 & 1010 & 1270 & 1360 \\
$\mathbf{8}$ & 1500 & 1500 & 1700 & 1810 & $\mathbf{1 8}$ & 720 & 840 & 1320 & 2040 \\
$\mathbf{9}$ & 850 & 1760 & 1360 & 1660 & $\mathbf{1 9}$ & 720 & 1240 & 1710 & 920 \\
$\mathbf{1 0}$ & 1610 & 1380 & 1240 & 140 & $\mathbf{2 0}$ & 580 & 1180 & 1530 & 320 \\
\hline
\end{tabular}

Table 2: Gross Requirement

The tentative MPS Solution is obtained by substituting the corresponding parameters into MPS Generator which employs equation 5 as basis of algorithm. The given data result in MPS solution whose overall degree of satisfaction is achieved at $\mu_{D}=0.8943$ with the achievement level of each objective as follow.

$$
\operatorname{EI}^{*}\left(Z_{1}\right)=1201 ; \operatorname{RNM}^{*}\left(Z_{2}\right)=160 ; \operatorname{BSS}^{*}\left(Z_{3}\right)=172 ; O C^{*}\left(Z_{4}\right)=240 \mathrm{~min}
$$

These achievement levels are regarded as planning goal that must be achieved as execution. Figure 4 depict the profile of load capacity distribution produced by MPS generator. It shows that the requirement capacities are not distributed equally among the available resources. Some resources are over-loaded, some of them have been fully occupied, and the rest are partly occupied (under-loaded). If the historical information about the potential uncertainty in the manufacturing system is known or foreeable, the distribution of load capacity may be adjusted accordingly so that its unexpected impact can be minimized.

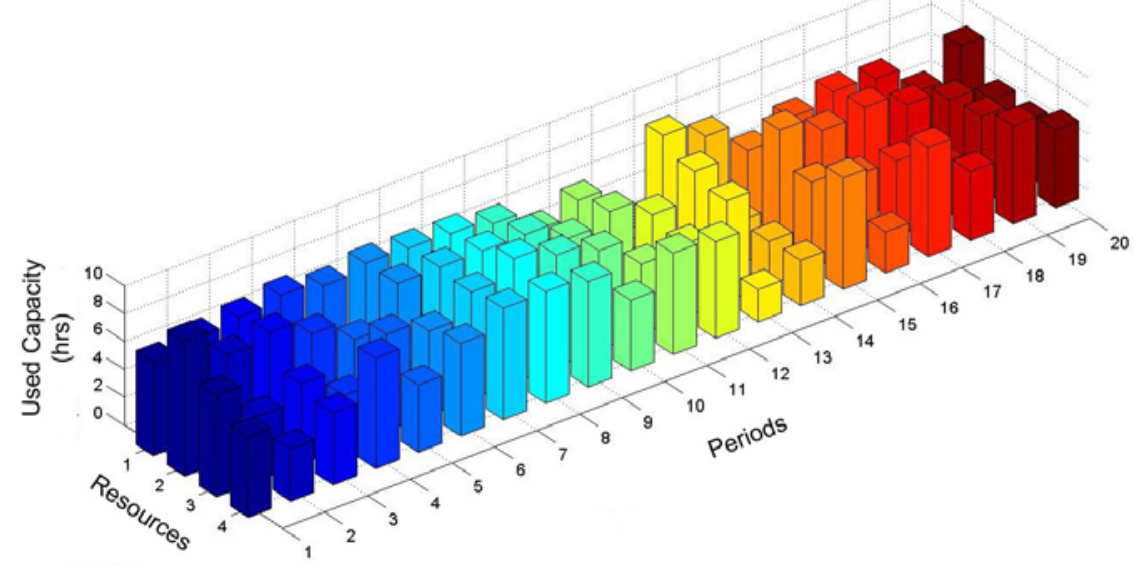

Figure 3 The profile of load capacity distribution

For the sake of simplicity, in this case the random machine breakdown (Table 3) and the varying processing time (Table 4) are chosen as the underlying cause of 
uncertainty. The processing time or production rate of each product is assumed as random normal distribution instead of constant numbers.

\begin{tabular}{c|c|c|c|c|c|c|c}
\hline $\begin{array}{l}\text { Failure } \\
\text { Control }\end{array}$ & $\begin{array}{c}\text { Prod. } \\
\text { Resource }\end{array}$ & $\begin{array}{c}\text { Stochastic } \\
\text { Distribution }\end{array}$ & \multirow{2}{*}{ Availability } & \multicolumn{2}{|c|}{ MTBF (min) } & \multicolumn{2}{c}{ MTTT (min) } \\
\cline { 5 - 7 } & & & Mean & Deviation & Mean & Deviation \\
\hline STO_1 & WCFXM01 & normal & $98 \%$ & 490 & 160 & 10.0 & 3.33 \\
STO_2 & WCFXM02 & normal & $96 \%$ & 300 & 100 & 12.5 & 4.20 \\
STO_3 & WCFXM03 & normal & $97 \%$ & 356 & 119 & 11.0 & 3.67 \\
STO_4 & WCFXM04 & normal & $95 \%$ & 238 & 79.2 & 12.5 & 4.20 \\
\hline
\end{tabular}

Table 3 Statistic of machine failure

\begin{tabular}{c|c|c|c|c|c|c|c|c}
\hline \multirow{2}{*}{ Items } & \multicolumn{2}{|c|}{ WCFXM01 } & \multicolumn{2}{c|}{ WCFXM02 } & \multicolumn{2}{c|}{ WCFXM03 } & \multicolumn{2}{c}{ WCFXM04 } \\
\cline { 2 - 8 } & Mean & Std & Mean & Std & Mean & Std & Mean & Std \\
\hline P-403 & 360 & 20 & 360 & 60 & 360 & 25 & 360 & 90 \\
P-404 & 240 & 20 & 240 & 60 & 240 & 25 & 240 & 90 \\
P-405 & 180 & 20 & 180 & 60 & 180 & 25 & 180 & 90 \\
P-406 & 90 & 20 & 90 & 60 & 90 & 25 & 90 & 90 \\
\hline
\end{tabular}

Table 4 Varying processing time (s/lot-size)

For analysis purpose, the simulation is carried out in several scenarios.

Scenario 1 No uncertainty or disturbance occurs during simulation

Scenario 2 Uncertainty is applied but no optimization is made

Scenario 3 Uncertainty is applied and adjustment is carried out by shifting overload to available resource at the same period, $p$.

Scenario 4 Uncertainty is applied and adjustment is carried out by shifting overload to available resource at period $p$ and $p-1$.

Scenario 5 Uncertainty is applied and adjustment is carried out by shifting overload to available resource at period $p, p-1$ and $p-2$.

Scenario 6 Uncertainty is applied and adjustment is carried out by shifting overload to available resource at period $p, p-1, p-2$ and $p-3$.

The simulation outputs for all scenarios are summarized in Table 5. In this case, DM's objectives are the only indicator used to justify the planning validity. Master schedule which is able to deliver DM goals is considered as final solution.

\begin{tabular}{c|c|c|l|c|c|c|c}
\hline No & Uncertainty & Scenario & \multicolumn{1}{|c|}{ Remark } & $\boldsymbol{E I}$ & $\boldsymbol{R N M}$ & $\boldsymbol{B S S}$ & $\boldsymbol{O C}(\boldsymbol{m i n})$ \\
\hline 1 & No & 1 & DM's Goal Satisfied & 1201 & 160 & 172 & 240 \\
2 & Yes & 2 & Not Yet Optimized & 1201 & 160 & 172 & 740 \\
3 & Yes & 3 & Optimization Stage 1 & 1201 & 160 & 172 & 304 \\
4 & Yes & 4 & Optimization Stage 2 & 1215 & 160 & 171 & 266 \\
5 & Yes & 5 & Optimization Stage 3 & 1261 & 160 & 169 & 88 \\
6 & Yes & 6 & Optimization Stage 4 & 1248 & 160 & 177 & 8 \\
\hline
\end{tabular}

Table 5 Simulation Output 


\section{$6 \quad$ Evaluation}

The first scenario is assumed as the ideal environment where no unexpected disturbance occurs. This simulation is meant to show that planning goals are easily obtained if there is no discrepancy between reality and schedule (all variables are deterministic). The uncertainty begins to be applied to the second scenario. The processing time is set random within certain deviation instead of constant and some machines suffer failures. One can see that DM's goals can no longer be met, which is indicated by the significant increasing of overtime, from 240 to $740 \mathrm{~min}$. It confirms that the tentative MPS turns into unrealistic due to uncertainty.

The third scenario, in which some load capacities are shifted to another resource at the same period, is able to almost half the unplanned overtime from 740 to $304 \mathrm{~min}$ without influencing other criteria. However, it does not meet DM's goals yet. The fourth scenario, in which some overload capacities is leveled to the previous period, can drop successfully the unplanned overtime close to the planned overtime while other criteria seem not to be changed. The fifth scenario and sixth scenario indicate the similar results, where the overtime can be forced down significantly but it bring implication to the increase of inventory level. This emphasizes that in the MPS problem, the inventory level and overtime are conflicting objectives.

As a whole, the result exhibits that shifting loads to early planning period is able to get MPS to be more realistic. In practice, it implies that one should manufacture products as earlier as possible to anticipate peak load in the coming period if the production system is quite unstable. This strategy may increase inventory but keep service level high. Among the given results, the fourth scenario is probably one which is the most approximate to DM's goals and may be chosen as final solution.

This simulation model considers a volatile manufacturing system which is indicated by frequent breakdown that occurs $1-2$ times a day. In such volatile environment, relying only on the reactive action during execution phase can lead to the poor resource allocation and causes much unnecessary delay. Therefore, a realistic and optimum schedule, which takes into account the prediction of breakdown in its planning phase, is inevitable. In this context, one can see that the prediction of uncertainty plays important role in MPS creation using this methodology. Fail to acquire accurately information about the dynamic behavior of manufacturing system may make the planning output even getting worse.

\section{Conclusion and Future Work}

Uncertainty is one of root causes of the production planning inaccuracy. This study has proved that the underlying causes of uncertainty such as varying processing time, machine failure, etc have considerable implication on the MPS. Ignoring them can lead to incorrect decision and ultimately make poor production and delivery performance. Through simulation approach, this study has been able to recognize 
the effect of uncertainty and subsequently attempts to diminish its effect to the master schedule.

However, it is acknowledged that the given model may be only a simplified reality of manufacturing system. More sophisticated model should be developed to examine further the reliability of proposed methodology. The underlying cause of uncertainty needs to be extent as well; not only involving random breakdown and stochastic processing time but also other unpredictable occasions due to limited buffer space, labor shortage, material shortage, etc. Moreover, the applicability of this methodology in various environments with different grade of uncertainty may be worthwhile to be investigated as well. Note that this methodology may be not considered for stable environment in which uncertainty are insignificant and ignorable.

\section{Variables}

$K \quad$ Total quantity of different products (SKU)

$R \quad$ Total quantity of different productive resources

$P \quad$ Total number of planning periods

TH Total planning horizon

$\mathrm{OH}_{k} \quad$ Initial available inventory (on-hand), at the first scheduling period

$G R_{k p} \quad$ Gross requirement for product $k$ at period $p$

$B S_{k p} \quad$ Standard lot size for product $k$ at period $p$

$N R_{k p} \quad$ Net requirement for product $k$ at period $p$, considering infinity capacity

$S S_{k p} \quad$ Safety inventory level for product $k$ at period $p$

$U R_{k p} \quad$ Production rate for product $k$ at resource $r$ (units per hour)

$A C_{k p} \quad$ Available capacity, in hours, at resource $r$ at period $p$

$B N_{k p r} \quad$ Quantity of standard lot sizes needed for the production of the product $k$ at resource $r$, at period $p$ (number of lots)

$M P S_{k p r} \quad$ Total quantity to be manufactured of product $k$ at resource $r$, period $p$

$M{ }^{2} T_{k p} \quad$ Total quantity to be manufactured of the product $k$ at resource, at period $p$ (considering all available resources)

$B I_{k p} \quad$ Initial inventory level of the product $k$ at period $p$

${ }_{C U H} \quad$ Capacity used from the resource $r$ at period $p$

$C U P_{r p} \quad$ Percent rate obtained from the relation of the number of hours consumed from the resource $r$ at period $p$, and the available number of hours to the same resource and period

$G R_{k p} \quad$ Gross requirement for product $k$ at period $p$

$R M_{k p} \quad$ Total requirements met for product $k$ at period $p$

$R M_{k p r} \quad$ Total requirements met for product $k$ at period $p$ at resource $r$

$R N M_{k p} \quad$ Requirements not met for product $k$ at period $p$

$R_{o} \quad$ Over-utilized or overload or overcapacity production resource

$R_{u} \quad$ Under-utilized or under-load production resource

$y_{k} \quad$ Quantity of product $k$ to be shifted from $R_{o}$ to $R_{u}$

$x_{k o} \quad$ Planned quantity of product $k$ to be manufactured in $R_{o}$

$U_{k u} \quad$ Average of production rate $R_{u}$ to produce product $k$ (unit/hrs)

$U_{k o} \quad$ Average of production rate $R_{o}$ to produce product $k$ (unit/hrs) 
$O L \quad$ Actual overtime occurred in $R_{o}$

$R C \quad$ Remain capacities in $R_{u}$

$S_{k u} \quad$ Percentage of potential defect product occurred in $R_{u}$ during operation execution

$R T \quad$ Amount of load capacity to be reduced from $R_{o}(\mathrm{~min})$

DI Total number of defect products

\section{Literature}

[Bel70] Bellman, R. E., Zadeh, L. A.: Decision-making in a fuzzy environment. Management Science, Vol. 17 No. 4, 1970

[Hei06] Heizer, J. H., Render, B.: Operations Management, Upper Saddle River, New York: Pearson Prentice Hall, 2006

[Hig92] Higgins, P., Browne, J.: Master production scheduling: a concurrent planning approach. Production Planning \& Control, Vol. 3, 1992

[Hop08] Hopp, W. J., Spearman, M. L.: Factory Physics, 3rd Edition, New York: McGraw Hill, 2008

[Kla96] Klafehn, K., Weinroth, J., Boronico, J.: Computer simulation in operation management, Westport: Greenwood Publishing Group, Inc, 1996

[Koc98] Kochhar, A. K., Ma, X., Khan, M. N.: Knowledge-based systems approach to the development of accurate and realistic master production schedules, Journal of Engineering Manufacture, Vol. 212 pp.453-60, 1998

[Koh05] Koh, S. L., Gunasekaran, A., Saad, S. M.: Tacking uncertainty in ERPcontrolled manucaturing environment: a knowledge management approach, International Journal Advanced Manufacturing Technology, DOI 10.1007/s00170-005-0251-2, 2005

[Moo05] Moon, Y. B., Phatak, D.: Enhancing ERP system's functionality with discrete event simulation, Industrial Management and Data System, Vol. 105 No. 9, pp.1206-1224, 2005

[Pro99] Proud, J. F.: Master Scheduling, 2rd Edition, John-Wile-Sons Inc, 1999

[Sak93] Sakawa, M.: Fuzzy sets and interactive multiobjective optimization, New York: Plenum Press, 1993

[Sap01] SAP IDES Documentation, Release 4.6C., Walldorf, Germany: SAP, 2001

[Sdz03] DOSIMIS-3 for MS-WINDOWS, Version 3.2, Dortmund: SDZ GmbH, 2003

[She03] Sheikh, K.: Manufacturing Resource Planning (MRP II), New York, USA: McGraw-Hill, 2003

[Soa08] Soares, M. M., Vieira, G. E.: A New multi-objective optimization method for master production scheduling problems based on genetic algorithm, International Journal of Advanced Manufacturing Technology, DOI 10.1007/s00170-008-1481-x, 2008

[Vie03] Vieira, G. E., Favaretto, F., Ribas, P. C.: Comparing genetic algorithms and simulated annealing in master production scheduling problems, Proceeding of 17th International Conference on Production Research, Blacksburg, Virginia, USA, 2003 
[Vie04] Vieira, G. E., Ribas, C. P.: A new multi-objective optimization method for master production scheduling problems using simulated annealing, International Journal of Production Research, Vol.42, 2004

[Zie93] Ziems, D., Neumann, G., Georgiev, M., Tolujev, J., Sujev, W.: Materialflußsimulation mit Dosimis-3, Magdeburg, 1993

[Zim78] Zimmermann, H. J.: Fuzzy programming and linear programming with several objective functions, Fuzzy Sets and Systems, 1, pp. 45-55, 1978 